\title{
Complex Tasks and Robots
}

\author{
A. Martinengo ${ }^{1}$, M. Campani ${ }^{2}$, and V. Torre ${ }^{1}$ \\ ${ }^{1}$ Dipartimento di Fisica and ${ }^{2}$ Consorzio I.N.F.M. \\ Via Dodecaneso 33, 16146 Genova, Italy
}

Robots have to move and survive in a variety of environments. In order to do so they have to avoid obstacles, find a collision free trajectory and cope with erroneous information; therefore, they must have rather efficient feedback loops, very similar to animal reflexes (Brooks, 1986). Real robots, however, have also to accomplish useful tasks, which are likely to have a reasonable degree of complexity. The purpose of this research is to perform experiments, in an unfriendly environment, with a robot having to accomplish tasks, which from a computational point of view may be rather complex, such as the exploration of a maze, the recovery of its structure and the planning of some nearly optimal trajectories through the maze. The robot used in the experimentation moves in a laboratory where a maze was constructed by sticking black stripes on the floor. The vision system of the robot is composed of a single uncalibrated T.V. camera pointing to the floor. In order to obtain almost real time performances, the perceptual world of the robot has been simplified, and is composed of black stripes and silhouettes over a flat floor with reflexes and shadows. A network of three computers is used to control the system behaviour. The processing of images is quite simple: the gray levels of the image are binarized, edges are extracted, and edge chains are approximated with a collection of straight segments. The robot has to recognize the direction of black stripes and the presence of junctions and silhouettes. The recognition of the silhouettes is based on the analysis of edge segments and takes into account the deformations due to perspective projection. In order to process the visual information correctly the system requires the setting of several parameters. By using an automatic learning procedure, given the lighting conditions, the material of the floor and of the black stripes, it was possible after a period of supervised learning to obtain a set of parameters, which could be used rather reliably. When the visual information has been processed, the robot has to move according to its task and its perception of the visual world. Two different strategies have been used: often, it is not necessary to follow the black stripes much carefully; in these cases the velocity of the wheels is continuously updated without halting the robot. In the presence of a junction, a better control is necessary: the robot has to reach a complete stop and adjust its position and heading direction according to its perception. During the navigation along a single stripe, the robot uses the first strategy; when a junction has been detected the robot switchs to the second. The main task concerns the exploration of the maze and the recovering of its structure, and has been implemented using a slightly modified version of the standard depth first search algorithm; by using this new strategy the number of edges that have to be followed to complete the exploration of the maze has been reduced. The experiments performed with standard low cost hardware show that the robot follows the lines of the maze in a crude way, but at a remarkable speed $(0.2$ meters $/ \mathrm{sec})$; moreover, since the vision system is almost uncalibrated, when the viewing camera is tilted from the nearly optimal position, the robot is still able to explore the maze, although with a more irregular trajectory. The robot is also able to accomplish its tasks, even if its wheels are maliciously slid or an erroneous stripe is shown for a while. Two factors contribute to this robust behaviour: the short time required for closing the optomotor feedback loop (200-500 msec) and a control strategy largely based on qualitative measurements. A way to evaluate the relative difficulty is to measure the time spent by the system solving different problems. Given the available computing power the robot spends a large amount of time in the control of its motion, and a significant fraction of the total time in the communication between the different computers. This time can be drastically reduced by a proper hardware mounted on board of the robot. The system will spend a significant fraction of the total time reasoning and planning only when the combinatorial complexity of the problem to be solved becomes relevant, for instance in the case of a very complex maze, composed of hundreds of junctions. In the presence of mazes with a limited number of junctions, the robot spends most of its time seeing and moving. These experiments suggest that the hard problem in the control of real robots, performing standard inspection procedures or monitoring, is perception and motor control. 